\title{
Both Poly(ethylene glycol) and Poly(methyl ethylene phosphate) Guide Oriented Adsorption of Specific Proteins
}

\author{
Christoph Bernhard, ${ }^{\dagger}$ Steven J. Roeters, ${ }^{\ddagger \oplus}$ Kristin N. Bauer, ${ }^{\dagger}$ Tobias Weidner, ${ }^{\ddagger \oplus}$ Mischa Bonn, ${ }^{\dagger}$
} Frederik R. Wurm, ${ }^{\dagger}$ and Grazia Gonella*, ${ }^{\dagger}$ (i)

\author{
${ }^{\dagger}$ Max Planck Institute for Polymer Research, Ackermannweg 10, 55128 Mainz, Germany \\ ${ }^{\ddagger}$ Department of Chemistry, Aarhus University, 8000 Aarhus C, Denmark
}

\section{Supporting Information}

\begin{abstract}
Developing new functional biomaterials requires the ability to simultaneously repel unwanted and guide wanted protein adsorption. Here, we systematically interrogate the factors determining the protein adsorption by comparing the behaviors of different polymeric surfaces, poly(ethylene glycol) and a poly(phosphoester), and five different natural proteins. Interestingly we observe that, at densities comparable to those used in nanocarrier functionalization, the same proteins are either adsorbed (fibrinogen, human serum albumin, and transferrin) or repelled (immunoglobulin $\mathrm{G}$ and lysozyme) by both polymers. However, when adsorption takes place, the specific surface dictates the amount and orientation of each protein.
\end{abstract}

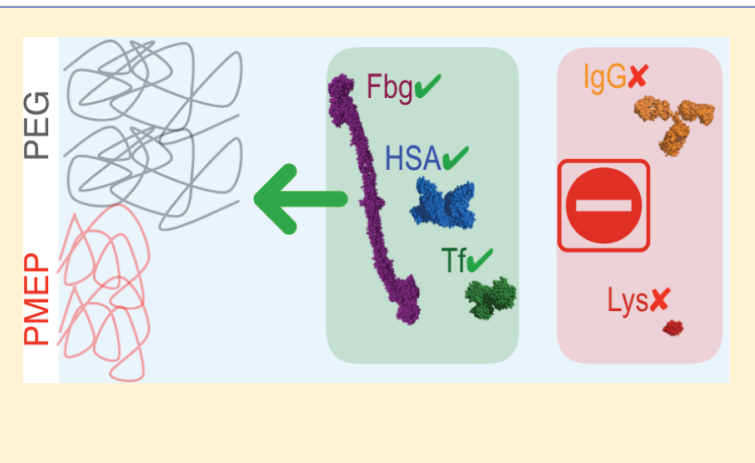

\section{INTRODUCTION}

The adsorption of biomolecules to specific surfaces is important for biomedical areas as diverse as replacement surgery, pathogen screening, biomolecular sensing, and drug delivery. The need to control, prevent, and/or sense the adsorption of biomolecules to the surface of materials has fueled synthetic efforts to find better materials capable of doing so. ${ }^{1}$ Different avenues can be followed to this end through the use of biological molecules (biocides), surface patterning (superhydrophobicity), or polymers characterized by high hydration or high exclusion volumes. ${ }^{2}$ In the biomedical field, nanoparticles have potential application as both drug nanocarriers and biosensors. ${ }^{3-6}$ However, despite their potential, both applications often suffer from non-specific protein adsorption at the surface of the nano-object. These nonspecifically bound proteins form the so-called protein corona, which can lead to blockage of the binding sites and, consequently, decrease the efficacy or sensitivity. ${ }^{7,8}$ Thus, a major challenge is the control of the surface properties. In particular, their optimization relies on two major factors: (1) promoting specific binding of the desired detection entity, with a preferential directionality, while (2) preventing non-specific protein adsorption.

On the one hand, specific binding can be favored by the use of both covalent and non-covalent immobilization of proteins at the surface of the biosensors. ${ }^{6,9,10}$ In addition to immobilization, the binding sites of the proteins need to be accessible for proper targeting. ${ }^{4,9,10}$ Thus, the orientation of the immobilized proteins at the surface is of paramount importance $^{4,9}$ as recently shown, by comparing nanoparticles covered by "favorably" oriented physisorbed proteins with "unfavorably" chemisorbed proteins. ${ }^{4,11}$ On the other hand, non-specific protein adsorption is commonly reduced by additional protein-repelling functionalization, for instance, using poly(ethylene glycol) (PEG). ${ }^{5,9,10,12}$ However, even though unspecific protein adsorption is reduced with PEG, it is known that the protein adsorption is not fully prevented at the surface of PEGylated nanoparticles. ${ }^{13}$ Furthermore, we have recently shown that the PEG packing density controls not only the quantity of adsorbed fibrinogen (Fbg) but also its orientation at the surface. ${ }^{14}$

Here, we compare the behavior of chemically different polymeric surfaces, PEG and a member of the poly(phosphoester) (PPE) family, namely, poly(methyl ethylene phosphate) (PMEP). In fact, PPEs have been established as good alternatives to PEG functionalization for nanoparticles to reduce protein adsorption. ${ }^{13,15}$ We have found that both PEG and PMEP seem to be unable to fully prevent Fbg and albumin adsorption, when used at surface densities commonly used in nanoparticle coatings, but rather promote the formation of a protein corona. ${ }^{14,16}$ More specifically, we compare and contrast PEG and PMEP in terms of their interactions with proteins (adsorption versus repulsion) and screen for different proteins to understand whether the protein repelling ability resides in the polymers or proteins.

\section{EXPERIMENTAL SECTION}

In our experiments, we use model systems consisting of polymers, modified with a long $\left(\mathrm{C}_{18}\right)$ alkyl chain to render them surface-active, so that monolayers can be formed at the air/aqueous phosphate-

Received: July 22, 2019

Revised: September 26, 2019

Published: September 30, 2019 
buffered saline (PBS) subphase. The non-ionic surfactants used in this work are schematically shown in panels $\mathrm{a}$ and $\mathrm{b}$ of Scheme 1 and are a

Scheme 1. Chemical Structures of (a) $C_{18}-\mathrm{PEG}$ and (b) $\mathrm{C}_{18^{-}}$ PMEP Surfactant and (c) Spacefill Representation of Fbg, HSA, Tf, IgG, and Lys from Left to Right ${ }^{a}$

a)<smiles>CCC(C)(C)COC(C)(C)CCOC(C)C</smiles>

b)

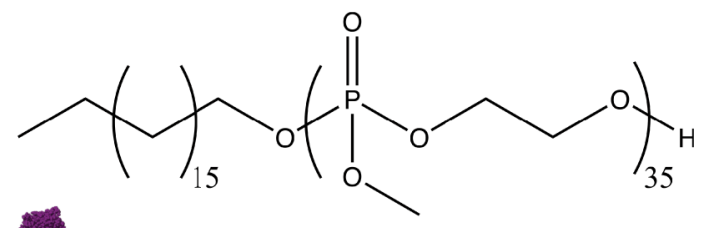

c)
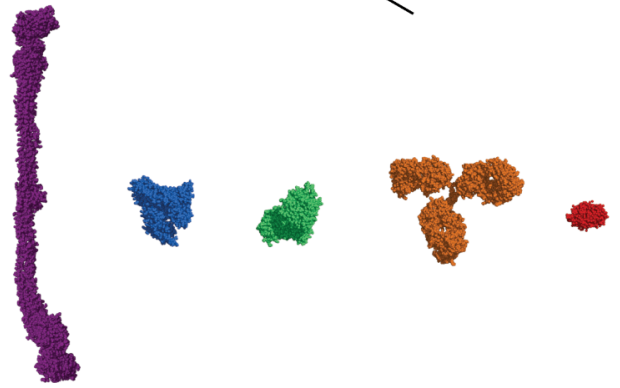

${ }^{a}$ See Table 1 for the PDB codes.

PEG derivative, Lutensol AT50 (BASF Germany), and a PMEP surfactant, which was synthesized and characterized according to refs 16-18, which will be referred to, in the remainder of this work, as $\mathrm{C}_{18}$-PEG and $\mathrm{C}_{18}$-PMEP, respectively. We use Brewster angle microscopy (BAM) (see section S1A of the Supporting Information) and vibrational sum-frequency generation (SFG) spectroscopy to study the conformation and hydration of the polymeric monolayers, as described in ref 16 , respectively. To investigate the surfactantprotein interactions, small volumes of concentrated human fibrinogen (Fbg), human serum albumin (HSA), transferrin (Tf), immunoglobulin G (IgG), or lysozyme from hen egg white (Lys) (Scheme 1c) solutions are injected into the subphase of the monolayers. The proteins represent a variety of natural proteins with different functions, sizes, and isoelectric points (see Table 1). Furthermore,

Table 1. Protein Characteristics

\begin{tabular}{ccccc} 
protein & $\begin{array}{c}\mathrm{MW}^{a} \\
(\mathrm{kDa})\end{array}$ & $\begin{array}{c}\text { isoelectric point } \\
(\mathrm{IEP})\end{array}$ & $\begin{array}{c}\text { estimated charge }{ }^{b} \text { at } \\
\mathrm{pH} 7.4\end{array}$ & $\begin{array}{c}\text { PDB } \\
\text { code }\end{array}$ \\
\hline Fbg & 325 & 5.5 & -15.2 & $3 \mathrm{GHG}$ \\
$\mathrm{HSA}$ & 65 & 4.8 & -16.1 & $1 \mathrm{AO6}$ \\
$\mathrm{Tf}$ & 75 & 6 & -3.0 & $2 \mathrm{HAV}$ \\
$\mathrm{IgG}$ & 150 & 7.5 & +0.2 & $1 \mathrm{IGT}$ \\
Lys & 15 & 11 & +7.3 & $1 \mathrm{DPX}$
\end{tabular}

${ }^{a}$ Approximated. ${ }^{b}$ Calculated with the Protein Calculator, version 3.4 (http://protcalc.sourceforge.net/).

we use a chemically modified cationic human serum albumin (cHSA), obtained as described in ref 19 (see also section S1B of the Supporting Information). To investigate the protein adsorption behavior onto the two different surfactant monolayers $\mathrm{C}_{18}$-PEG and $\mathrm{C}_{18}$-PMEP, we combine surface pressure measurements and SFG. The surface pressure measurements are sensitive to the amount of adsorbed protein at the interface, while SFG spectroscopy is a secondorder nonlinear optical spectroscopy, which is surface-selective as a result of symmetry selection rules and has been frequently used to obtain information on the conformation and orientation of proteins at interfaces. ${ }^{20-28}$ More details about the sample preparation and the experiments can be found below and in section S1 of the Supporting Information.

Monolayer Preparation and Surface Pressure Measurements. The $\mathrm{C}_{18}$-PMEP surfactant and $\mathrm{C}_{18}$-PEG are dissolved in a 9:1 (v/v) mixture of high-purity chloroform (VWR Chemicals) and methanol (VWR Chemicals) to obtain solutions with $50 \mu \mathrm{M}$ concentration. Subsequently, the surfactants are spread on a PBS ( $\mathrm{pH} 7.4$, Sigma-Aldrich) subphase. The mean molecular area per surfactant at the surface for all measurements is $\sim 5 \mathrm{~nm}^{2}$ and was calculated by tracking the number of molecules present at the surface and by knowing the total area available to them. For the protein adsorption studies, $\sim 100 \mu \mathrm{L}$ of highly concentrated solutions of either Fbg (Sigma-Aldrich), HSA (Sigma-Aldrich), Tf (Sigma-Aldrich), IgG (antibodies-online $\mathrm{GmbH}$ ) or Lys (Roche) dissolved in PBS buffer solution is injected into the subphase to reach a final protein concentration of $\sim 0.1 \mathrm{mg} / \mathrm{mL}$. The proteins and PBS tablets are used as received. During the SFG experiments, the surface pressure is simultaneously monitored during all measurements using a DeltaPi tensiometer (Kibron, Finland).

Vibrational SFG Spectroscopy. For our experiments, we use a SFG setup in reflection geometry to measure the amide I and $\mathrm{OH}$ stretch signals at the monolayer/PBS interface in the absence and presence of proteins in the subphase. For the SFG process, we overlap a spectrally tunable broadband femtosecond infrared (IR) pulse in space and time with a spectrally narrow visible (VIS) pulse. The visible pulses at $800 \mathrm{~nm}$ wavelength ( $\sim 40 \mathrm{fs}$ pulse duration) are generated by a regenerative Ti:sapphire amplifier (Spitfire Ace, Spectra Physics, Santa Clara, CA, U.S.A.) with a repetition rate of 1 $\mathrm{kHz}$. A beam splitter divides the VIS beam into two parts. One of them is spectrally narrowed by an etalon (SLS Optics, Ltd.) to a full width at half maximum (fwhm) of $\sim 15 \mathrm{~cm}^{-1}$. The pulse energy of the VIS after the etalon is $\sim 20 \mu \mathrm{J}$. The other part of the VIS beam is used to pump an optical parametric amplifier (TOPAS-C, Spectra Physics, Santa Clara, CA, U.S.A.), which employs an additional difference frequency generation scheme to generate the broadband tunable IR pulses. The IR pulse energies within this work are in the range of $\sim 2.5-5 \mu \mathrm{J}$. Then, both beams are focused onto the monolayer/PBS interface, and the generated SFG signal is collimated with a lens, dispersed with a spectrograph (Acton SpectraPro 300i, Princeton Instruments, Trenton, NJ, U.S.A.), and finally collected with an electron-multiplied charge-coupled device camera (Newton EMCCD 971P-BV, Andor Technology, Ltd., U.K.). The polarization state of IR, VIS, and SFG beam is controlled by polarizers and half-wave plates in the setup. The experimental stage is flushed with nitrogen during all measurements in the amide $\mathrm{I}$ and free $\mathrm{OH}$ region, to suppress IR absorption from water in the atmosphere. The monolayers are spread on the PBS subphase in a homemade circular Teflon-coated trough (diameter, $\sim 8 \mathrm{~cm}$; volume, $\sim 20 \mathrm{~mL}$ ). The trough is rotated during the measurements to prevent laser-heatinginduced displacement of the surfactants. ${ }^{29}$ Unless otherwise stated, all spectra are collected in the ssp (s-polarized SFG signal, s-polarized VIS beam, and p-polarized IR beam) polarization combination. The average accumulation time for one SFG spectra is $10 \mathrm{~min}$. Afterward, the spectra are background-subtracted and normalized to account for the spectral shape of the IR beam with a non-resonant reference signal from z-cut quartz. The room temperature for all measurements is controlled to be $(22 \pm 1){ }^{\circ} \mathrm{C}$.

\section{RESULTS AND DISCUSSION}

We prepare monolayers of $\mathrm{C}_{18}$-PEG and $\mathrm{C}_{18}$-PMEP on a PBS subphase using a mean molecular area of $5 \mathrm{~nm}^{2}$, comparable to the area per polymeric chain used at the surface of nanoparticles. ${ }^{13,15}$ Notwithstanding the differences in their chemical structures, in terms of their conformation and hydration, the two guiding parameters determining the protein-repelling ability of the polymers, ${ }^{30-34}$ PEG and PMEP, seem to be quite similar. In fact, characterization of the monolayers via BAM (ref 16 and sections S1A and S2A of 
the Supporting Information) suggests that, while the $\mathrm{C}_{18}$-PEG monolayer appears thicker than the monolayer formed by $\mathrm{C}_{18^{-}}$ PMEP and even though the packing density for both polymers is expected to be beyond the mushroom-to-brush conformational transition, they are unlikely in a fully extended brush conformation. In fact, in a previous study comparing different PPEs at the same surface density used in this work, PMEP seemed to adopt a more mushroom-like conformation compared to other PPEs. ${ }^{16}$ Also in terms of hydration, the two monolayers show similar characteristics (see section S2B of the Supporting Information for more details). If the polymer parameters would be the only factor determining the adsorption/repulsion of the proteins then, on the basis of the above considerations, one would expect that the two polymers would similarly adsorb or repel indiscriminately all proteins. To verify such a hypothesis, we inject small volumes of different protein solutions into the subphase.

Adsorption of Specific Proteins. Figure 1 shows the time evolution of the surface pressure at the $\mathrm{C}_{18}$-PMEP and $\mathrm{C}_{18^{-}}$

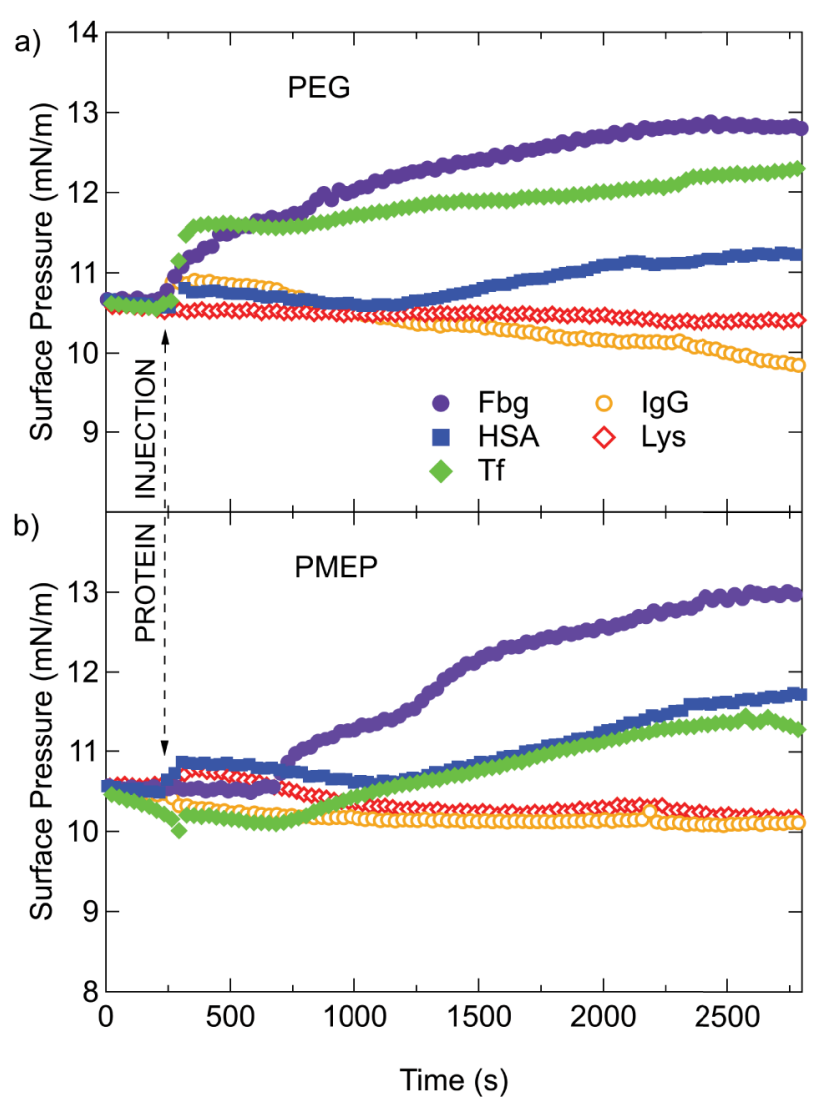

Figure 1. Surface pressure of the (a) $\mathrm{C}_{18}$-PEG and (b) $\mathrm{C}_{18}$-PMEP monolayers on PBS after injection of the Fbg (full purple circles), HSA (full blue squares), Tf (full green diamonds), IgG (empty orange circles), and Lys (empty red diamonds) solutions into the subphase at time $t \sim 250 \mathrm{~s}$. The mean molecular area for both monolayers is 5 $\mathrm{nm}^{2}$.

PEG monolayers upon protein injection. Fbg, HSA, or Tf induce an increase in the surface pressure over time, suggesting adsorption of these proteins onto both $\mathrm{C}_{18}$-PMEP and $\mathrm{C}_{18^{-}}$ PEG monolayers. Furthermore, IgG and Lys seem to be repelled by both PEG and PMEP because no increase in the surface pressure is observed in the $45 \mathrm{~min}$ following the injection. Thus, the ability to adsorb or repel the protein, in this case, does not seem polymer-specific but rather proteinspecific. The results obtained on $\mathrm{C}_{18}$-PEG monolayers are in line with those observed for nanoparticles, where enrichment in the protein corona of some plasma proteins was observed. ${ }^{13}$ This agreement validates our strategy to model the nanoparticle surface with a planar polymer-aqueous solution interface.

Indeed, various properties of both surface and protein are responsible for their interaction, and they can be divided into geometrical, chemical, and electrical interactions. From the protein side, the protein size can be an important factor, because bigger proteins have, in principle, the ability to form more contacts with the surface and small proteins have the possibly to interdigitate within the polymeric coating; another factor is the sequence of amino acids, because charged amino acids are more amphiphilic and, therefore, usually located on the surface of the protein and, thus, more readily available to interact with the surface. Of course, also, the amino acid sequence, directly affecting the distribution of charges, in the protein has an impact, and proteins close to their isoelectric point might be able to adsorb in higher quantities because of the reduction of the electrostatic interprotein repulsion. On the surface side, relevant properties for the interaction with the protein include the roughness, because it can favor interactions with the proteins by increasing the effective surface area, the chemical composition that determines which groups are available for interaction, and finally, the presence of charges or dipoles at the surface and ions in the aqueous solution that modify the liquid phase near the surface and, in turn, affect the interaction between the protein and the surface. ${ }^{35} \mathrm{We}$ attempted to rationalize the commonalities between adsorbed and repelled proteins and set out to check whether size and/or charge could be the determining factors. However, in our experiments, we observe that the smallest protein (Lys) is repelled, whereas the largest protein (Fbg) adsorbs, and no pattern based on size can be highlighted because the second biggest (IgG) and second smallest (HSA) protein invert such a trend. A parameter that better correlates with protein adsorption seems to be the isoelectric point (IEP); negatively charged proteins (IEP < 7.4: Fbg, HSA, and Tf) adsorb, while positively charged proteins (IEP > 7.4: IgG and Lys) are repelled. To further test this hypothesis while keeping other parameters constant, we use a chemically modified cHSA. ${ }^{19}$ With this modification, the overall changes to the system are minimal, because only the protein surface is changed. Figure 2 shows the surface pressure measurements after injection of cHSA into the subphase of the two different monolayers. As seen, the positively charged cHSA adsorbs onto both monolayers. Thus, also, the protein charge does not seem to be the driving factor in protein adsorption onto PEG and PMEP.

The fact that protein adsorption is very complex does not allow us to rule out other contributing factors, such as the structural rigidity of the proteins or finer properties, such as the charge and/or the hydrophobicity/hydrophilicity distribution of specific protein domains, and further investigation on such parameters is needed. What we can conclude at this stage is that, independent of the underlying reason, both surfaces present adsorption of specific proteins, which is the sought after property in the development of biomaterials. It is worth noting that the change in pressure induced by cHSA is bigger than that for HSA: $\sim 9$-fold on $\mathrm{C}_{18}$-PEG and $\sim 6$-fold for $\mathrm{C}_{18^{-}}$ PMEP. This might suggest a higher affinity of cHSA for the 


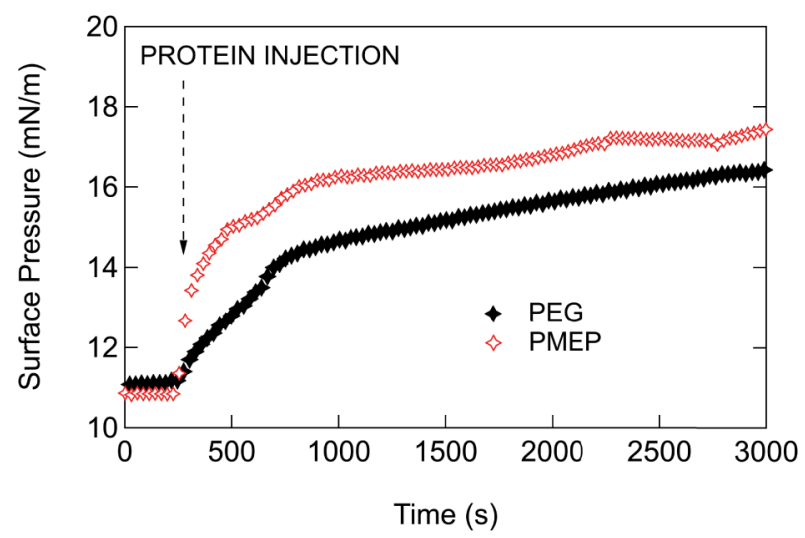

Figure 2. Surface pressure of $\mathrm{C}_{18^{-}}$PEG (full black stars) and $\mathrm{C}_{18^{-}}$ PMEP (empty red stars) monolayers on PBS after injection of the cHSA solution into the subphase at time $t \sim 250 \mathrm{~s}$. The mean molecular area for both monolayers is $5 \mathrm{~nm}^{2}$.

two polymeric surfaces. However, because the surface and the molecular weight of the two proteins are different, the adsorbed amounts cannot be directly obtained from the surface pressure measurement.

Amount and Orientation of the Adsorbed Protein. From Figure 1, it is clear that there is a difference in the affinity of HSA and Tf toward PEG and PMEP and that such a difference is not observed for Fbg. Now, we will investigate whether there is also a difference in the orientation induced by these two surfaces on the adsorbed proteins. We do so by collecting the SFG spectra in the amide I region after injection of the proteins in the subphase of the two monolayers.

Figure 3 shows the SFG spectra before (black) and after (colored) injection of either Fbg, HSA, or Tf into the subphase of the $\mathrm{C}_{18}$-PEG and $\mathrm{C}_{18}$-PMEP monolayers, respectively. Here, the general behavior of the proteins at the two different surfaces is very similar. Without proteins present in the subphase, no distinct peak is present in the amide I region between 1600 and $1700 \mathrm{~cm}^{-1}$ for either monolayer. In the
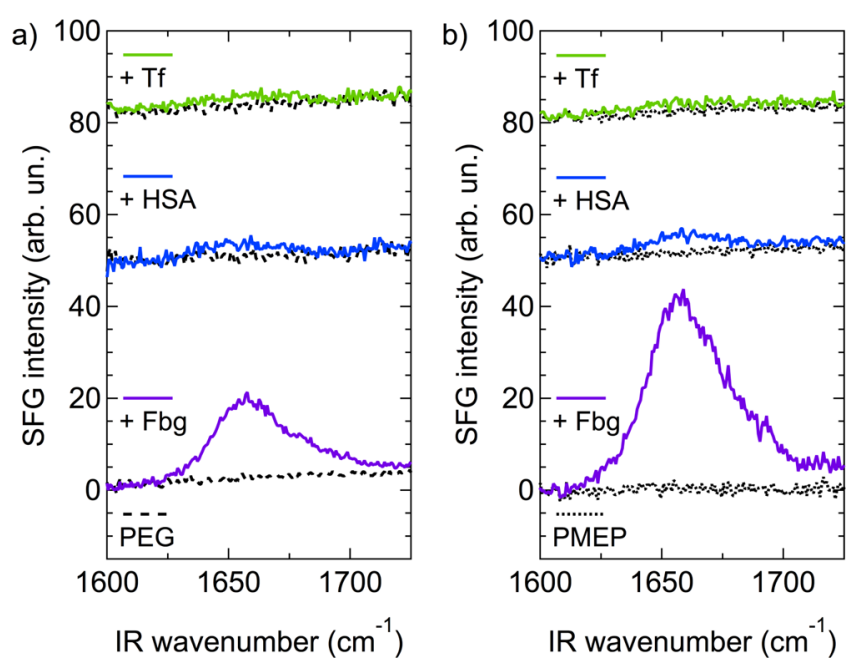

Figure 3. SFG spectra in the amide I region for the (a) $\mathrm{C}_{18}$-PEG and (b) $\mathrm{C}_{18}$-PMEP monolayers in the absence (discontinuous black lines) and presence of $0.1 \mathrm{mg} / \mathrm{mL} \mathrm{Fbg} \mathrm{(continuous} \mathrm{purple} \mathrm{line),} \mathrm{HSA}$ (continuous blue line), and $\mathrm{Tf}$ (continuous green line) in the subphase. The curves for HSA and Tf are offset vertically for clarity. The mean molecular area for both monolayers is $5 \mathrm{~nm}^{2}$. presence of Fbg, HSA, and Tf (see Figure S5 of the Supporting Information for a zoom-in of the spectra), a broad peak around $1660 \mathrm{~cm}^{-1}$ appears, which is assigned to $\alpha$-helices in the secondary structure of the proteins. Additionally, in the case of Fbg, a shoulder at around $1690 \mathrm{~cm}^{-1}$ is present, which is assigned to $\beta$-sheet structures. ${ }^{16}$ The presence of the amide I peak in the SFG spectra indicates that Fbg, HSA, and Tf not only adsorb onto the polymers but do so in an ordered manner.

Moreover, despite the thus far observed similarities in their behavior toward protein adsorption, PMEP and PEG present a distinct difference in the overall SFG signal intensity in the amide I region generated by HSA and Fbg. This can have several origins, namely, differences in the (1) amount of adsorbed protein, (2) protein orientation, and (3) protein conformation at the two surfaces or any combination of the factors mentioned above. In the case of HSA and Tf, it seems likely that differences in the amount of adsorbed protein are responsible for the differences in the SFG intensities, as also observed in the surface pressure measurements. For Fbg, on the other hand, the initial surface pressure is comparable for $\mathrm{C}_{18}$-PEG and $\mathrm{C}_{18}$-PMEP $(\sim 10.5 \mathrm{mN} / \mathrm{m})$, as is the relative change $(\sim 2.5 \mathrm{mN} / \mathrm{m})$. This suggests that similar amounts of Fbg adsorb onto both surfaces. Consequently, we conclude that the differences in the SFG signal intensities can only be explained by different orientation and/or conformation of Fbg on the two surfaces. Previous studies on the Fbg adsorption on other hydrophilic surfaces showed that Fbg can adopt various conformations upon adsorption. ${ }^{36-39}$ However, the most common conformations in all of these studies show a structure very close to the crystalline structure. Moreover, changes in the secondary structure of Fbg upon adsorption onto the polymer surfaces should be reflected in changes of the spectral shape of the amide I SFG signal. This is not observed in our study, where the normalized SFG spectra of Fbg adsorbed onto $\mathrm{C}_{18^{-}}$ PEG and $\mathrm{C}_{18}$-PMEP have the same shape (see Figure $S 6$ of the Supporting Information). We thus assume in the following that the Fbg conformation is mainly preserved upon adsorption on the two surfaces.

Insights into the protein orientation at the surface can be obtained through the collection of SFG spectra in different polarization combinations, ${ }^{40,41}$ even more so when this is combined with calculated spectra. ${ }^{42,43}$ Details on the calculation of the SFG spectra can be found in section S3 of the Supporting Information. Figure 4 shows the experimental and calculated SFG spectra in the amide I region for $\mathrm{Fbg}$ adsorbed on $\mathrm{C}_{18}$-PEG and $\mathrm{C}_{18}$-PMEP in ssp, ppp, and sps polarization combinations, respectively. The calculated SFG spectra (on the basis of the PDB structure) reproduce the spectral shape and center position of the experimental peaks very well, consistently with a preserved protein structure upon adsorption. Moreover, the fitted tilt angles of Fbg with respect to the surface plane indicate that the orientation of $\mathrm{Fbg}$ is different for the two surfaces: $\sim 33$ and 0 degrees for the $\mathrm{C}_{18^{-}}$ PEG and $\mathrm{C}_{18}$-PMEP monolayers, respectively (see section S3 of the Supporting Information). The change in surface pressure and the comparison of the SFG data and calculations indicate that, in the case of $\mathrm{C}_{18}$-PMEP, Fbg adsorbs parallel to the surface and affects the surface pressure because it "pushes" against the monolayer, while in the case of $\mathrm{C}_{18}$-PEG, Fbg is more likely to intercalate with the polymer. Therefore, we conclude that PEG and PMEP do adsorb Fbg in similar amounts but induce different orientations of this protein at the 

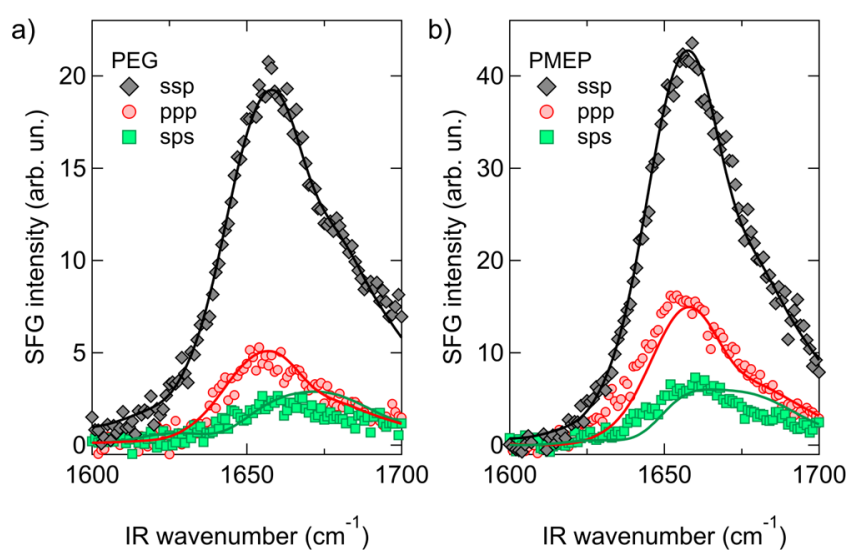

Figure 4. SFG spectra in the amide I region of $0.1 \mathrm{mg} / \mathrm{mL}$ Fbg at the (a) $\mathrm{C}_{18}$-PEG and (b) $\mathrm{C}_{18}$-PMEP monolayers in ssp (black diamonds), ppp (red circles), and sps (green squares) polarization combinations. The full lines represent the calculated SFG spectra for Fbg at the $\mathrm{C}_{18^{-}}$ PEG and $\mathrm{C}_{18}$-PMEP monolayers, respectively. The mean molecular area for both monolayers is $5 \mathrm{~nm}^{2}$.

surface for densities of the polymers comparable to those used to coat nanoparticles. Indeed, this result is not unexpected because the two polymers also orient water to different extents (see section S2B of the Supporting Information).

\section{CONCLUSION}

In conclusion, our results suggest that, for the proteins tested in this work, at surface densities relevant in nanoparticle functionalization, the two polymers of interest, PEG and PMEP, are able to adsorb specific proteins, meaning that proteins are not adsorbed or repelled indiscriminately, but also the two polymers adsorb or repel the same proteins. Furthermore, the specific polymeric surface affects the amount of adsorbed protein, such as in the case of HSA and Tf, or the protein orientation, as observed for Fbg. This finding is important for the development of biomaterial surfaces, because it shows that a single surface functionalization can be sufficient to control the quantity and orientation of specific proteins upon adsorption, while at the same time, it can suppress unspecific adsorption of others.

\section{ASSOCIATED CONTENT}

\section{S Supporting Information}

The Supporting Information is available free of charge on the ACS Publications website at DOI: 10.1021/acs.langmuir.9b02275.

Materials and methods, supporting measurements, and calculation of vibrational SFG spectra (PDF)

\section{AUTHOR INFORMATION}

\section{Corresponding Author}

*E-mail: gonella@mpip-mainz.mpg.de.

\section{ORCID $\odot$}

Christoph Bernhard: 0000-0001-6136-7461

Steven J. Roeters: 0000-0003-3238-2181

Tobias Weidner: 0000-0002-7083-7004

Mischa Bonn: 0000-0001-6851-8453

Frederik R. Wurm: 0000-0002-6955-8489

Grazia Gonella: 0000-0002-0470-4043

\section{Notes}

The authors declare no competing financial interest.

\section{ACKNOWLEDGMENTS}

The authors thank Seah Ling Kuan and Michael Müller for providing the cHSA. Furthermore, the authors thank Sudipta Das, Maksim Grechko, Christian Malm, Jan Schaefer, and Giovanni Settanni for fruitful discussions. Christoph Bernhard acknowledges support from the Max Planck Graduate Center with the Johannes Gutenberg University Mainz through a Ph.D. fellowship. Steven J. Roeters and Tobias Weidner acknowledge funding from the Villum Foundation and the Aarhus University Research Foundation (AUFF).

\section{REFERENCES}

(1) Morsbach, S.; Gonella, G.; Mailänder, V.; Wegner, S.; Wu, S.; Weidner, T.; Berger, R.; Koynov, K.; Vollmer, D.; Encinas, N.; Kuan, S. L.; Bereau, T.; Kremer, K.; Weil, T.; Bonn, M.; Butt, H.-J.; Landfester, K. Engineering Proteins at Interfaces: From Complementary Characterization to Material Surfaces with Designed Functions. Angew. Chem., Int. Ed. 2018, 57, 12626-12648.

(2) Antifouling Surfaces and Materials: From Land to Marine Environment; Zhou, F., Ed.; Springer: Berlin, Germany, 2015; DOI: $10.1007 / 978-3-662-45204-2$.

(3) Kirsch, J.; Siltanen, C.; Zhou, Q.; Revzin, A.; Simonian, A. Biosensor technology: Recent advances in threat agent detection and medicine. Chem. Soc. Rev. 2013, 42, 8733.

(4) Trilling, A. K.; Beekwilder, J.; Zuilhof, H. Antibody orientation on biosensor surfaces: A minireview. Analyst 2013, 138, 1619-1627.

(5) Howes, P. D.; Chandrawati, R.; Stevens, M. M. Colloidal nanoparticles as advanced biological sensors. Science 2014, 346, 1247390-1247390.

(6) Bhakta, S. A.; Evans, E.; Benavidez, T. E.; Garcia, C. D. Protein adsorption onto nanomaterials for the development of biosensors and analytical devices: A review. Anal. Chim. Acta 2015, 872, 7-25.

(7) Cedervall, T.; Lynch, I.; Lindman, S.; Berggård, T.; Thulin, E.; Nilsson, H.; Dawson, K. A.; Linse, S. Understanding the nanoparticleprotein corona using methods to quantify exchange rates and affinities of proteins for nanoparticles. Proc. Natl. Acad. Sci. U. S. A. 2007, 104, 2050-2055.

(8) Lynch, I.; Dawson, K. A. Protein-nanoparticle interactions. Nano Today 2008, 3, 40-47.

(9) Lin, P. C.; Weinrich, D.; Waldmann, H. Protein biochips: Oriented surface immobilization of proteins. Macromol. Chem. Phys. 2010, 211, 136-144.

(10) Jonkheijm, P.; Weinrich, D.; Schröder, H.; Niemeyer, C. M.; Waldmann, H. Chemical strategies for generating protein biochips. Angew. Chem., Int. Ed. 2008, 47, 9618-9647.

(11) Tonigold, M.; Simon, J.; Estupiñán, D.; Kokkinopoulou, M.; Reinholz, J.; Kintzel, U.; Kaltbeitzel, A.; Renz, P.; Domogalla, M. P.; Steinbrink, K.; Lieberwirth, I.; Crespy, D.; Landfester, K.; Mailänder, V. Pre-adsorption of antibodies enables targeting of nanocarriers despite a biomolecular corona. Nat. Nanotechnol. 2018, 13, 862-869.

(12) Blankenburg, J.; Maciol, K.; Hahn, C.; Frey, H. Poly(ethylene glycol) with Multiple Aldehyde Functionalities Opens up a Rich and Versatile Post-Polymerization Chemistry. Macromolecules 2019, 52, 1785-1793.

(13) Schöttler, S.; Becker, G.; Winzen, S.; Steinbach, T.; Mohr, K.; Landfester, K.; Mailänder, V.; Wurm, F. R. Protein adsorption is required for stealth effect of poly(ethylene glycol)- and poly(phosphoester)-coated nanocarriers. Nat. Nanotechnol. 2016, 11, 372-377.

(14) Bernhard, C.; Roeters, S. J.; Franz, J.; Weidner, T.; Bonn, M.; Gonella, G. Repelling and ordering: The influence of poly(ethylene glycol) on protein adsorption. Phys. Chem. Chem. Phys. 2017, 19, 28182-28188. 
(15) Simon, J.; Wolf, T.; Klein, K.; Landfester, K.; Wurm, F. R.; Mailänder, V. Hydrophilicity Regulates the Stealth Properties of Polyphosphoester-Coated Nanocarriers. Angew. Chem., Int. Ed. 2018, $57,5548-5553$

(16) Bernhard, C.; Bauer, K. N.; Bonn, M.; Wurm, F. R.; Gonella, G. Interfacial Conformation of Hydrophilic Polyphosphoesters Affects Blood Protein Adsorption. ACS Appl. Mater. Interfaces 2019, 11, $1624-1629$

(17) Wolf, T.; Steinbach, T.; Wurm, F. R. A Library of Well-Defined and Water-Soluble Poly(alkyl phosphonate)s with Adjustable Hydrolysis. Macromolecules 2015, 48, 3853-3863.

(18) Bauer, K. N.; Liu, L.; Andrienko, D.; Wagner, M.; Macdonald, E. K.; Shaver, M. P.; Wurm, F. R. Polymerizing Phostones: A Fast Way to In-Chain Poly(phosphonate)s with Adjustable Hydrophilicity. Macromolecules 2018, 51, 1272-1279.

(19) Eisele, K.; Gropeanu, R. A.; Zehendner, C. M.; Rouhanipour, A.; Ramanathan, A.; Mihov, G.; Koynov, K.; Kuhlmann, C. R.; Vasudevan, S. G.; Luhmann, H. J.; Weil, T. Fine-tuning DNA/ albumin polyelectrolyte interactions to produce the efficient transfection agent cBSA-147. Biomaterials 2010, 31, 8789-8801.

(20) Wang, J.; Even, M. A.; Chen, X.; Schmaier, A. H.; Waite, J. H.; Chen, Z. Detection of Amide I Signals of Interfacial Proteins in Situ Using SFG. J. Am. Chem. Soc. 2003, 125, 9914-9915.

(21) Chen, X.; Wang, J.; Sniadecki, J. J.; Even, M. A.; Chen, Z. Probing $\alpha$-Helical and $\beta$-Sheet Structures of Peptides at Solid/Liquid Interfaces with SFG. Langmuir 2005, 21, 2662-2664.

(22) Haupert, L. M.; Simpson, G. J. Chirality in Nonlinear Optics. Annu. Rev. Phys. Chem. 2009, 60, 345-365.

(23) Yan, E.; Ding, Y.; Chen, C.; Li, R.; Hu, Y.; Jiang, X. Polymer/ silica hybrid hollow nanospheres with $\mathrm{pH}$-sensitive drug release in physiological and intracellular environments. Chem. Commun. 2009, 2718

(24) Braunschweig, B.; Mukherjee, P.; Kutz, R. B.; Rumpel, A.; Engelhardt, K.; Peukert, W.; Dlott, D. D.; Wieckowski, A. Spectroscopy of Electrified Interfaces with Broadband Sum Frequency Generation: From Electrocatalysis to Protein Foams. In Vibrational Spectroscopy at Electrified Interfaces; Wieckowski, A., Braunschweig, B., Korzeniewski, C., Eds.; John Wiley \& Sons, Inc.: Hoboken, NJ, 2013; Chapter 4, pp 120-150, DOI: 10.1002/9781118658871.ch4.

(25) Weidner, T.; Castner, D. G. SFG analysis of surface bound proteins: A route towards structure determination. Phys. Chem. Chem. Phys. 2013, 15, 12516

(26) Roy, S.; Covert, P. A.; FitzGerald, W. R.; Hore, D. K. Biomolecular Structure at Solid-Liquid Interfaces As Revealed by Nonlinear Optical Spectroscopy. Chem. Rev. 2014, 114, 8388-8415.

(27) Yan, E. C. Y.; Fu, L.; Wang, Z.; Liu, W. Biological Macromolecules at Interfaces Probed by Chiral Vibrational Sum Frequency Generation Spectroscopy. Chem. Rev. 2014, 114, 84718498.

(28) Ding, B.; Jasensky, J.; Li, Y.; Chen, Z. Engineering and Characterization of Peptides and Proteins at Surfaces and Interfaces: A Case Study in Surface-Sensitive Vibrational Spectroscopy. Acc. Chem. Res. 2016, 49, 1149-1157.

(29) Backus, E. H. G.; Bonn, D.; Cantin, S.; Roke, S.; Bonn, M. Laser-Heating-Induced Displacement of Surfactants on the Water Surface. J. Phys. Chem. B 2012, 116, 2703-2712.

(30) Jeon, S. I.; Lee, J. H.; Andrade, J. D.; De Gennes, P. G. ProteinSurface Interactions in the Presence of Polyethylene Oxide. J. Colloid Interface Sci. 1991, 142, 149-158.

(31) Marsh, D.; Bartucci, R.; Sportelli, L. Lipid membranes with grafted polymers: Physicochemical aspects. Biochim. Biophys. Acta, Biomembr. 2003, 1615, 33-59.

(32) Chen, S.; Li, L.; Zhao, C.; Zheng, J. Surface hydration: Principles and applications toward low-fouling/nonfouling biomaterials. Polymer 2010, 51, 5283-5293.

(33) Schwierz, N.; Horinek, D.; Liese, S.; Pirzer, T.; Balzer, B. N.; Hugel, T.; Netz, R. R. On the relationship between peptide adsorption resistance and surface contact angle: A combined experimental and simulation single-molecule study. J. Am. Chem. Soc. 2012, 134, 19628-19638.

(34) Barrett, A.; Imbrogno, J.; Belfort, G.; Petersen, P. B. Phosphate Ions Affect the Water Structure at Functionalized Membrane Surfaces. Langmuir 2016, 32, 9074-9082.

(35) Dee, K. C; Puleo, D. A.; Bizios, R. An Introduction to TissueBiomaterial Interactions; Wiley: New York, 2002; DOI: 10.1002/ 0471270598.

(36) Köhler, S.; Schmid, F.; Settanni, G. The Internal Dynamics of Fibrinogen and Its Implications for Coagulation and Adsorption. PLoS Comput. Biol. 2015, 11, e1004346.

(37) Köhler, S.; Schmid, F.; Settanni, G. Molecular Dynamics Simulations of the Initial Adsorption Stages of Fibrinogen on Mica and Graphite Surfaces. Langmuir 2015, 31, 13180-13190.

(38) Protopopova, A. D.; Barinov, N. A.; Zavyalova, E. G.; Kopylov, A. M.; Sergienko, V. I.; Klinov, D. V. Visualization of fibrinogen $\alpha \mathrm{C}$ regions and their arrangement during fibrin network formation by high-resolution AFM. J. Thromb. Haemostasis 2015, 13, 570-579.

(39) Clarke, M. L.; Wang, J.; Chen, Z. Conformational Changes of Fibrinogen after Adsorption. J. Phys. Chem. B 2005, 109, 2202722035.

(40) Zhuang, X.; Miranda, P.; Kim, D.; Shen, Y. Mapping molecular orientation and conformation at interfaces by surface nonlinear optics. Phys. Rev. B: Condens. Matter Mater. Phys. 1999, 59, 12632-12640.

(41) Nguyen, K. T.; Le Clair, S. V.; Ye, S.; Chen, Z. Orientation Determination of Protein Helical Secondary Structures Using Linear and Nonlinear Vibrational Spectroscopy. J. Phys. Chem. B 2009, 113, 12169-12180.

(42) Roeters, S. J.; Van Dijk, C. N.; Torres-Knoop, A.; Backus, E. H. G.; Campen, R. K.; Bonn, M.; Woutersen, S. Determining in situ protein conformation and orientation from the amide-I sumfrequency generation spectrum: Theory and experiment. J. Phys. Chem. A 2013, 117, 6311-6322.

(43) Schmüser, L.; Roeters, S.; Lutz, H.; Woutersen, S.; Bonn, M.; Weidner, T. Determination of Absolute Orientation of Protein $\alpha$ Helices at Interfaces Using Phase-Resolved Sum Frequency Generation Spectroscopy. J. Phys. Chem. Lett. 2017, 8, 3101-3105. 